\title{
Novel design procedure for microwave filters
}

\section{Guglielmi, Member IEEE, and A. Alvarez Melcon}

European Space Research and Technology Centre (ESTEC) P.O. Box 299, Noordwijk, The Netherlands

Fax: 31-1719-84596. Tel: 31-1719-83493

\section{Abstract}

In this paper a novel design procedure is described for the hardware implementation of microwave filters. The procedure is based on a very accurate and efficient software package for the full-wave simulation of the structure and consists of a step-by-step procedure that systematically leads to dimensioning of the complete filter geometry. Following the procedure described, each successive step only involves the dimensioning of a maximum of four physical parameters. As an illustrative example, the design of a nine pole non-uniform filter is discussed indicating how the procedure is indeed very efficient.

\section{Summary}

The design of a microwave filter usually starts with the selection of a suitable transfer function to satisfy a set of given electrical specifications. The next step is to go from the transfer function to an ideal electrical network representation involving, for instance, lengths of transmission line and impedance inverters. The final step is the transition from the ideal network elements to real waveguide components that, once assembled together, exhibit the required electrical behavior. In this paper we focus on this last step, describing a novel step-by-step procedure for the transition from an ideal network to the actual waveguide hardware. This novel procedure is based on the use of a very accurate and fast software package for the analysis of waveguide filters based on thick inductive windows called WIND developed at ESTEC [1]. The key feature of the procedure is that each step of the design has a very clear goal and only involves the optimization of a limited number of physical parameters thereby greatly simplifying the design task.

\section{Application}

To illustrate the novel approach, we describe the design of a non-uniform nine pole Chebycheff filter in rectangular waveguide. The initial steps in the design have been carried out following the well known procedure described in [2], leading to the ideal network representation shown in Fig. 1. The ideal line parameters and impedance inverter values are given in Tab. 1 . Note that the structure is non-uniform in that each resonator is implemented with a transmission line with different characteristic impedance (this choice was dictated by the original out-of-band requirements for the filter).

Once the ideal network is obtained, the first step of the novel procedure can be carried out. It consists of computing the $S_{21}$ parameter in $\mathrm{dB}$ of the single first cavity of the ideal network in a doubly terminated configuration obtaining the response shown in Fig. 2 with a thick line. The next step is the simulation with WIND of only the first cavity of the real waveguide structure with the width adjusted to give the required line impedance. The goal is to recover the performance of the ideal resonator and involves the adjustment (software or soft tuning) of only three parameters, namely the resonator length and the input and output aperture widths. The optimized result is shown by the thin line in Fig. 2 . The next step is the computation of the transmission characteristics of the first two ideal cavities obtaining the result shown with a thick line in Fig. 3. The soft tuning procedure is now repeated on the real structure using WIND in order to recover the ideal behavior. In this step the soft tuning involves only four parameters, namely the additional aperture and line length, and the output aperture of the first cavity together with the first cavity length. This is due to the interactions between adjacent cavities. However, the line length and output aperture of the first cavity usually require only very minor adjustments. The optimized results are shown again in Fig. 3 with the thin line.

The procedure can now continue with the addition of the third cavity. Note that, from now on, any successive step always involves the soft tuning of only four physical parameters, namely the additional line length and output aperture, and the line length and output aperture of the previous cavity.

The design continues up to the fifth cavity and is then repeated in the opposite direction also up to the fifth cavity. This is necessary because the filter being designed is not symmetric.

The final results obtained are shown by the thin line in Fig. 4. The thick line represents the ideal response of the network in Fig. 1. As we can see, the ideal response has been essentially recovered. Table 2 gives the final dimensions of the complete filter. A prototype is now being manufactured and measured results will be presented during the talk.

\section{Conclusions}

In this paper, a novel step-by-step procedure is presented which allows for the systematic design of microwave filter. Each step of the procedure has a simple goal and involves the adjustment of a maximum of four physical parameters irrespective of the complexity of the final filter structure. The procedure is based on the availability of full-wave simulation software and can in principle be applied to any type of microwave filter.

\section{References}

[1] M. Guglielmi, G. Gheri, M. Calamia, G. Pelosi, "Rigorous Multimode Network/Numerical Representation of Inductive Step," Accepted for publication in IEEE, Microwave Theory and Techniques, July 1992.

[2] G. Matthaei, L. Young and M.T. Jones, Microwave Filters, Impedance-Matching Networks, and Coupling Structures, Artech House, Inc., 1985.

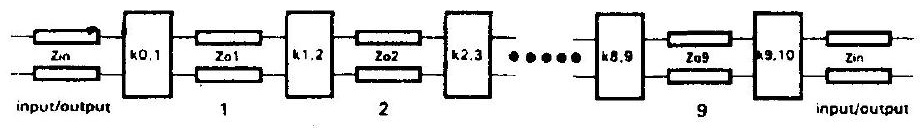

Fig. I Ideal network representation for a nine pole filter The length of the resonators is half wavelength at the center frequency.

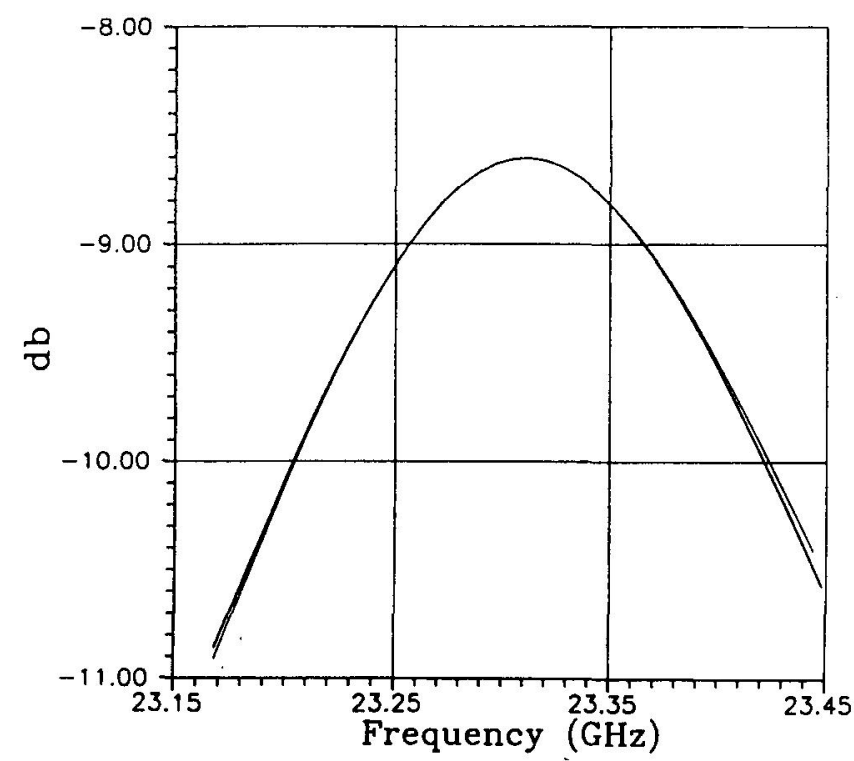

Fig. 2 Transmission characteristics of the first cavity of the filter in Fig. 1 


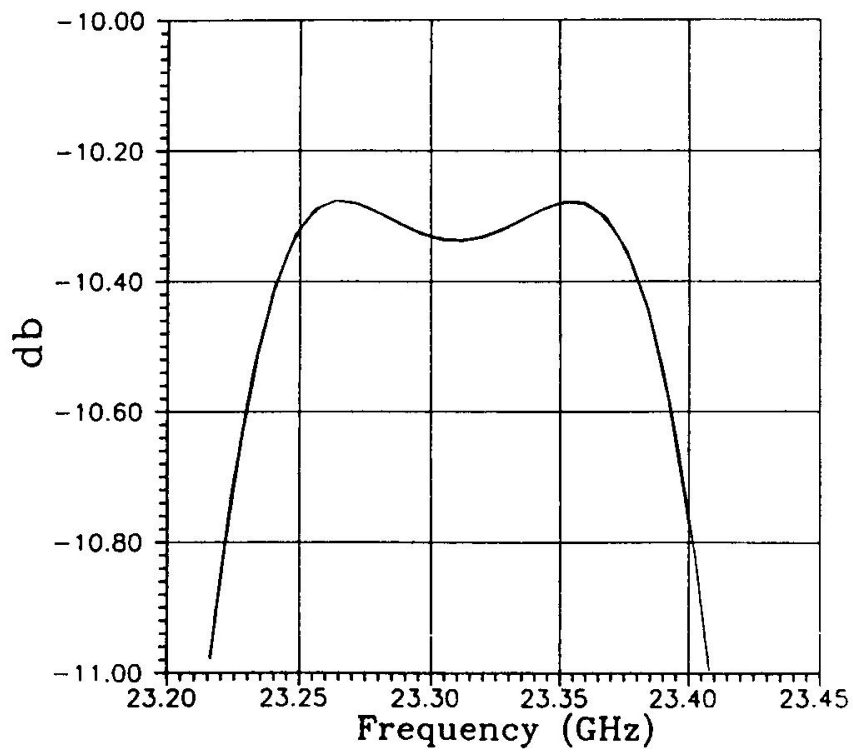

Fig. 3 Transmission characteristics of the first two cavities of the filter in Fig. !.

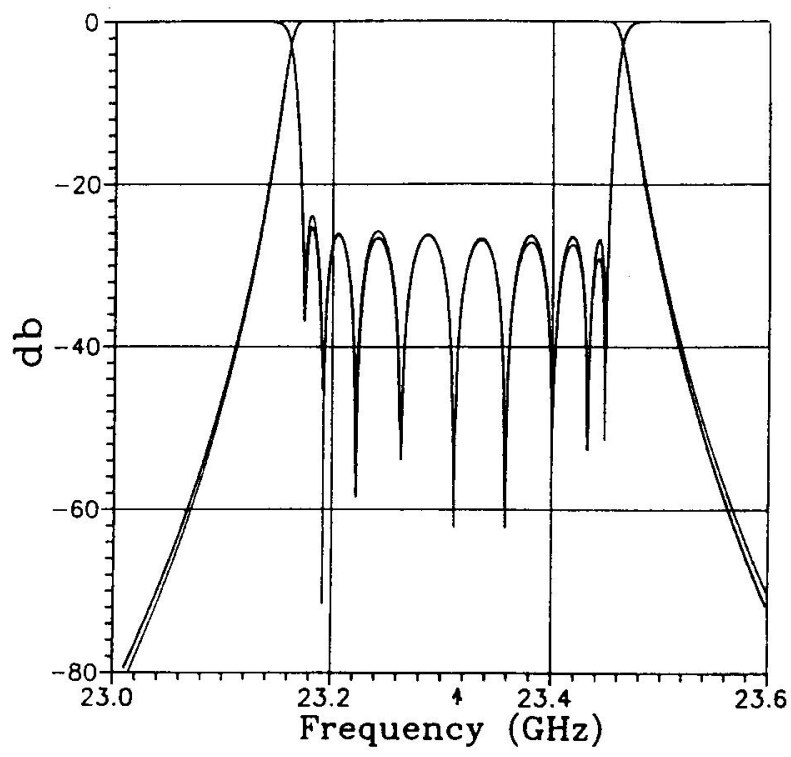

Fig. 4 Electrical characteristics of the complete filter.

\begin{tabular}{|ll|}
\hline \multicolumn{2}{|l|}{$\begin{array}{l}\text { Characteristic } \\
\text { Impedance } \\
\text { (ohms) }\end{array}$} \\
\hline Zin & 472.169 \\
Zo1 & 474.217 \\
Zo2 & 479.671 \\
Zo3 & 485.659 \\
Zo4 & 492.263 \\
Zo5 & 512.153 \\
Zo6 & 538.830 \\
Zo7 & 576.462 \\
Z08 & 633.705 \\
Zo9 & 732.486 \\
\hline
\end{tabular}

\begin{tabular}{|ll|}
\hline \multicolumn{2}{|l|}{$\begin{array}{l}\text { Impedance } \\
\text { Inverters } \\
\text { (ohms) }\end{array}$} \\
\hline k0,1 & 90.244 \\
$k 1.2$ & 13.255 \\
$k 2,3$ & 9.229 \\
$k 3,4$ & 8.759 \\
$k 4,5$ & 9.230 \\
$k 5,6$ & 10.570 \\
$k 6,7$ & 12.972 \\
$k 7,8$ & 18.124 \\
$k 8,9$ & 38.641 \\
$k 9,10$ & 173.242 \\
\hline
\end{tabular}

Tab. I Values of the elements of the ideal filter in Fig. 1.

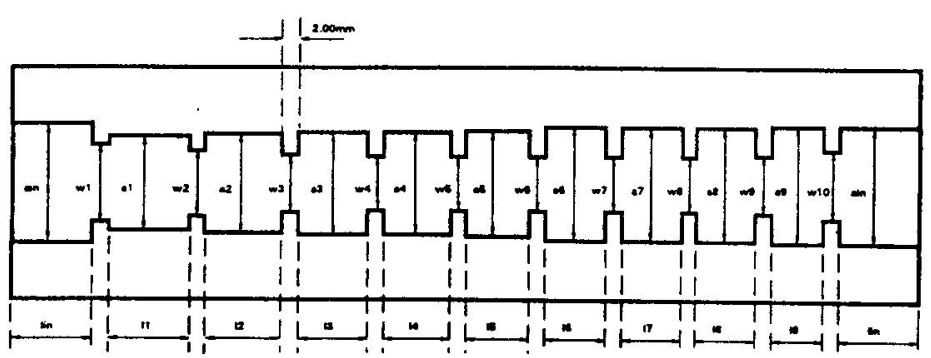

\begin{tabular}{|c|c|}
\hline \multicolumn{2}{|c|}{$\begin{array}{l}\text { Cavity } \\
\text { Widths } \\
\text { (mm) }\end{array}$} \\
\hline ain & 10.68 \\
\hline a 1 & 7.50 \\
\hline 82 & 8.00 \\
\hline 83 & 8.50 \\
\hline a4 & 9.00 \\
\hline a5 & 9.50 \\
\hline a 6 & 10.00 \\
\hline 87 & 10.20 \\
\hline a8 & 10.40 \\
\hline a9 & 10.60 \\
\hline
\end{tabular}

\begin{tabular}{|c|c|}
\hline \multicolumn{2}{|c|}{$\begin{array}{l}\text { Aperture } \\
\text { Widths } \\
\text { (mm) }\end{array}$} \\
\hline w1 & 5.364 \\
\hline w2 & 3.675 \\
\hline w3 & 3.183 \\
\hline w4 & 3.010 \\
\hline w5 & 2.924 \\
\hline w6 & 2.879 \\
\hline$w 7$ & 2.872 \\
\hline w8 & 2.919 \\
\hline w9 & 3.181 \\
\hline w10 & 4.964 \\
\hline
\end{tabular}

\begin{tabular}{|cc|}
\hline \multicolumn{2}{|l|}{$\begin{array}{l}\text { Cavity } \\
\text { Lengths } \\
\text { (mm) }\end{array}$} \\
\hline $\operatorname{lin}$ & 10.00 \\
11 & 9.818 \\
12 & 9.663 \\
13 & 8.998 \\
14 & 8.478 \\
15 & 8.094 \\
16 & 7.800 \\
17 & 7.689 \\
18 & 7.523 \\
19 & 6.678 \\
\hline
\end{tabular}

Tab. 2 Physical dimensions of the nine pole filter in Fig. 4. 